University of Nebraska - Lincoln

DigitalCommons@University of Nebraska - Lincoln

2-1986

\title{
Angular and Energy Dependence of Cross Sections for Ejection of Electrons from Water Vapor. III. 20- 150-keV Neutral-Hydrogen Impact
}

\author{
M. A. Bolorizadeh \\ University of Nebraska - Lincoln \\ M. Eugene Rudd \\ University of Nebraska - Lincoln, erudd@unl.edu
}

Follow this and additional works at: https://digitalcommons.unl.edu/physicsrudd

Part of the Physics Commons

Bolorizadeh, M. A. and Rudd, M. Eugene, "Angular and Energy Dependence of Cross Sections for Ejection of Electrons from Water Vapor. III. 20- 150-keV Neutral-Hydrogen Impact" (1986). M. Eugene Rudd Publications. 8.

https://digitalcommons.unl.edu/physicsrudd/8

This Article is brought to you for free and open access by the Research Papers in Physics and Astronomy at DigitalCommons@University of Nebraska - Lincoln. It has been accepted for inclusion in M. Eugene Rudd Publications by an authorized administrator of DigitalCommons@University of Nebraska - Lincoln. 


\title{
Angular and energy dependence of cross sections for ejection of electrons from water vapor. III. $20-150-\mathrm{keV}$ neutral-hydrogen impact
}

\author{
M. A. Bolorizadeh* and M. E. Rudd \\ University of Nebraska-Lincoln, Lincoln, Nebraska 68588-0111
}

(Received 20 May 1985)

\begin{abstract}
Absolute values of cross sections for electron production in collisions of neutral hydrogen atoms of 20-150 keV energy with water vapor molecules have been measured as a function of the ejection energy and angle. The range of angles was $10^{\circ}$ to $160^{\circ}$ and the electron energy range was $1-300 \mathrm{eV}$. The doubly differential cross sections were integrated over angle and/or energy to obtain singly differential cross sections, total electron production cross sections, and average energies of ejection. The angular distribution of the electron loss peak was found to have some features in common with the cross section for elastic scattering of electrons.
\end{abstract}

\section{INTRODUCTION}

While there have been several measurements of doubly differential cross sections (DDCS) for electron ejection by proton and other charged-particle impacts on various target gases, the only DDCS available for neutral impact over a wide range of projectile and electron energies and emission angles are for $15-150 \mathrm{keV}$ hydrogen atoms on helium. ${ }^{1}$ Even total electron-production cross-section measurements for neutral-hydrogen impacts are available for only a very few target gases. ${ }^{2}$ Duncan and Menendez ${ }^{3}$ have studied emission of electrons from $\mathrm{H}^{0}$ and $\mathrm{H}^{-}$collisions with argon and Meckbach et al. ${ }^{4}$ have presented measurements on the $\mathrm{H}^{0}+\mathrm{He}$ system.

At least in part because of the lack of experimental data with which to compare, there has been little theoretical work done on neutral-impact ionization. Bates and Griffing ${ }^{5}$ calculated energy distributions of electrons from hydrogen atoms of four different energies incident on atomic hydrogen and Dalgarno and Griffing ${ }^{6}$ calculated DDCS for the same collision partners at 10,100 , and $1000 \mathrm{keV}$ using the Born approximation. No calculations are known for any other targets.

Measurements of DDCS involving projectile ions carrying one or more electrons ${ }^{7,8}$ have shown a large peak at an electron velocity equal to the projectile velocity due to electron loss from the projectile. Our $\mathbf{H}^{0}+\mathrm{He}$ studies ${ }^{1}$ showed that the same peak is present with neutral impacts. In the present work we have also studied this peak and determined its dependence on beam energy and ejection angle.

Since water is an important constituent of the atmosphere and a major component of living cells, collision cross section data on it is of special interest in several applied areas. As protons of a few 10's of $\mathrm{keV}$ in the solar wind precipitate through the atmosphere a large fraction of them are neutralized. Thus any study of energy deposition in the atmosphere must take account of ionization by neutral-hydrogen atoms. For the same reason, radiation damage studies in cells must be based on information on neutral as well as charged-particle interactions.

To our knowledge there is neither experimental nor theoretical data on ionization of water molecules by neutral-hydrogen impact. The present study provides both differential and total ionization cross sections for the energy range of $20-150 \mathrm{keV}$. A comparison is made between the DDCS for neutral-hydrogen, proton, and electron impact to provide a basis for understanding the mechanisms of ionization.

\section{EXPERIMENTAL METHOD}

The apparatus used in this experiment has been described previously. ${ }^{1}$ The accelerator, collision chamber, electrostatic analyzer, and target-gas handling system are also described in the preceding paper.'

Nitrogen was used as the neutralizing gas in a chargetransfer cell in the beam line. As before, a potential of 250 to $750 \mathrm{~V}$ on the deflection plates following the charge-transfer cell eliminated the charged components from the beam. The electric field between the plates also served as a quenching field for metastable atoms and an ionizing field for Rydberg atoms as discussed earlier. ${ }^{1}$ Two different constantan foils of thicknesses 0.010 and $0.014 \mathrm{~mm}$ were used in the thermal detector for the beam. These gave sensitivities of 0.067 and $0.020 \mathrm{~V} / \mathrm{W}$, respectively. The sensitivities were measured with a proton beam, comparing the thermal detector output with the current to the detector connected as a Faraday cup. Because the sensitivity depended somewhat on the focusing of the beam, it was measured separately for each run.

The uncertainty in reading the collected beam charge varied from $4 \%$ at the higher beam energies to $7 \%$ at the lowest energy. Combined with the $7 \%$ uncertainty in measuring the target-gas pressure and the $8 \%$ uncertainty in the efficiency of the electron detector, this yields a total uncertainty of $11-13 \%$ in the measured DDCS with additional uncertainties at the extremes of the electronenergy range. The cross sections integrated over electron energy are estimated to have an uncertainty of $20 \%$.

\section{EXPERIMENTAL RESULTS}

Figure 1 shows the dependence of the DDCS for 150 $\mathrm{keV}$ impacts on electron energy for various angles. As in 


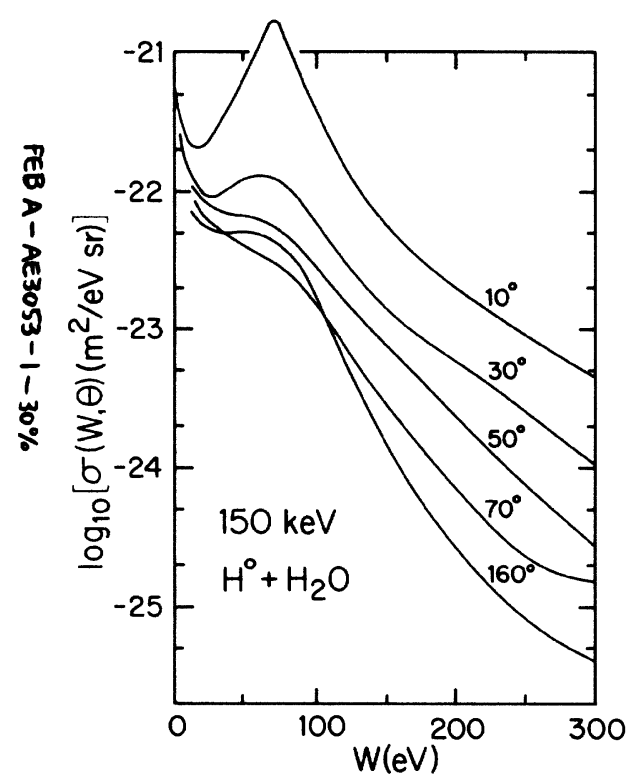

FIG. 1. Doubly differential cross sections for ejection of electrons by $150-\mathrm{keV} \mathrm{H}^{0}$ on water vapor vs energy of ejection at various angles.

the earlier work on helium, ${ }^{1}$ a dominating feature of the curves is the large peak at the energy corresponding to an electron velocity equal to the projectile velocity, in this case $82 \mathrm{eV}$. Although it comes at the same energy as the peak due to continuum charge transfer, it is not confined to the forward direction, and is quite prominent even at $160^{\circ}$. This peak is due to the detachment and subsequent elastic scattering of the electrons carried by the projectiles. This process, known as electron loss to the continuum (ELC), was first noted by Wilson and Toburen ${ }^{7}$ with $\mathrm{H}_{2}{ }^{+}$

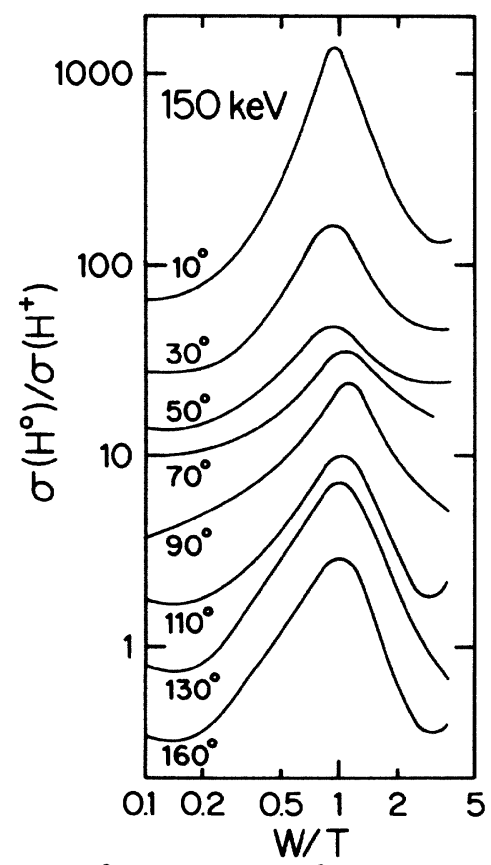

FIG. 2. Ratios of cross sections for $150-\mathrm{keV} \mathrm{H}^{0}$ to $\mathrm{H}^{+}$impacts vs ejection energy at various angles. The curves have been displaced in the vertical direction by arbitrary amounts for clarity.

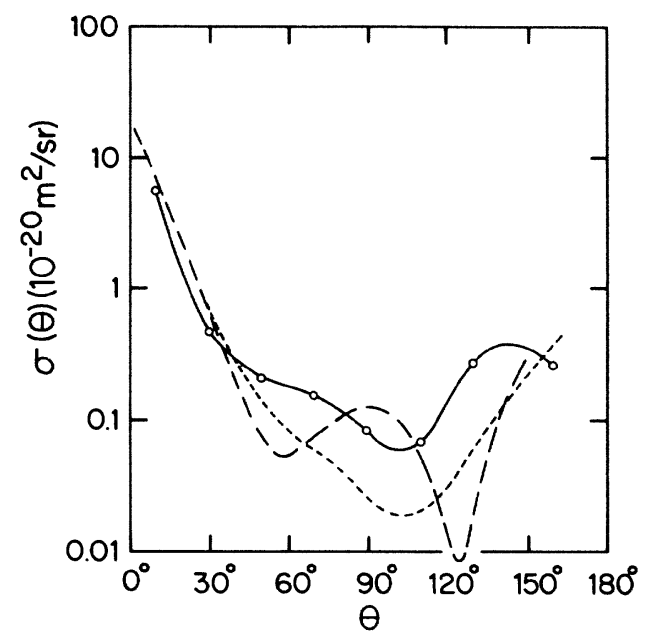

FIG. 3. Angular distribution of the area under the ELC peak for $150-\mathrm{keV} \mathrm{H}^{0}+\mathrm{H}_{2} \mathrm{O}$, points and solid line. Also shown is the same quantity for $500-\mathrm{keV} \mathrm{H}^{-}+$Ar from Ref. 8 , shown as the short-dashed curve, and the elastic differential cross sections for 100-eV $e^{-}+$Ar from Ref. 9, shown as the long-dashed curve.

projectiles.

To show this feature more clearly, these cross sections have been divided by the corresponding $\mathrm{H}^{+}$cross sections ${ }^{9}$ and plotted versus $\log (W / T)$ in Fig. $2 . \quad W$ is the ejected electron energy and $T=m_{e} v_{p}^{2} / 2$ where $m_{e}$ is the electron mass and $v_{p}$ is the projectile velocity. While the peak is not symmetric on this scale, it is approximately so when plotted versus $(W / T)^{1 / 2}$. It is seen that the relative size of the ELC peak is large at the small and large angles and smaller at intermediate angles.

To obtain absolute values of the cross section for this process versus angle, an integration was made over the peak. As a correction for the background of continuum

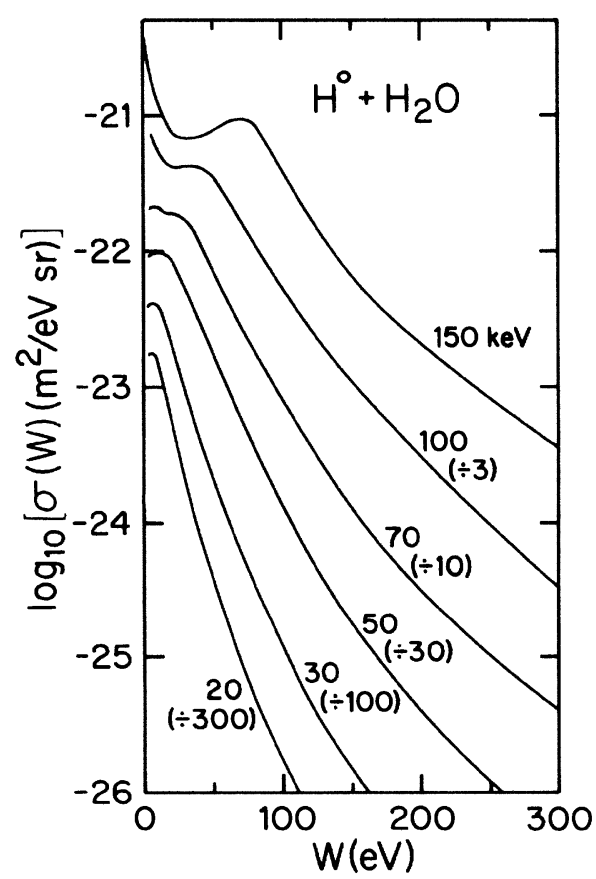

FIG. 4. Cross sections integrated over angles for $\mathrm{H}^{0}+\mathrm{H}_{2} \mathrm{O}$ vs electron-ejection energy. 


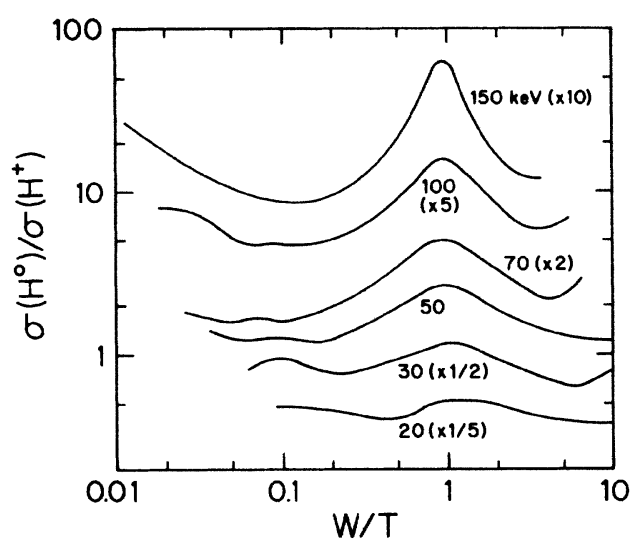

FIG. 5. Ratios of cross sections, integrated over angle, for $\mathbf{H}^{0}$ to $\mathrm{H}^{+}$impacts on water vapor.

electrons under the peak, the corresponding $\mathrm{H}^{+}$cross sections were also integrated and subtracted from the $\mathbf{H}^{0}$ values. Figure 3 shows the integrals over the peak obtained in this way plotted as a function of angle. Also shown are the cross sections for ELC for $500 \mathrm{keV}$ $\mathrm{H}^{-}+\mathrm{Ar}$ obtained by Duncan and $\mathrm{Menendez}^{3}$ and the elastic differential scattering cross sections for $100-\mathrm{eV}$ electrons on Ar from DuBois and Rudd. ${ }^{10}$ Even though the projectiles, targets, and energies are different for these three cases, there are definite points of similarity between the curves suggesting similar origins. Kövér et al. ${ }^{11}$ have made a study of the angular dependence of the ELC peak in $\mathrm{He}^{+}+\mathrm{Ar}$ and $\mathrm{H}_{2}{ }^{+}+\mathrm{Ar}$ collisions which also showed a rise in the backward direction.

The DDCS were integrated over the entire angular range to obtain singly differential cross sections (SDCS) at each value of $W$. A graph of the SDCS for various impact energies is given in Fig. 4. The ELC peak moves, as expected, to a lower energy as the impact energy decreases and it also becomes less prominent. Figure 5 shows the ratio of the SDCS for $\mathrm{H}^{0}$ to that of $\mathrm{H}^{+}$as a function of energy. Table I gives the SDCS for selected electron energies. By numerical integration of the SDCS, the total

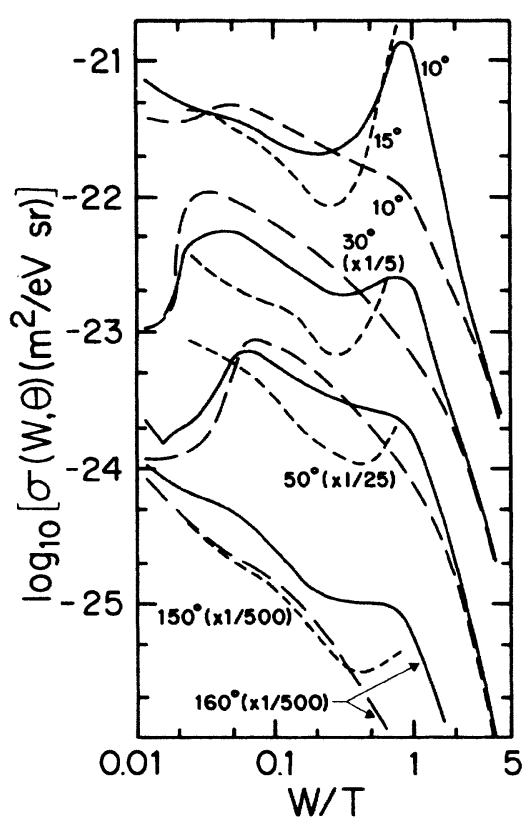

FIG. 6. Comparison of cross sections for electron production for various projectiles on water vapor. $150-\mathrm{keV} \mathrm{H}^{0}$, solid line; 150-keV H${ }^{+}$, long-dashed line; 80-eV $e^{-}$, short-dashed line.

electron production cross sections were obtained as well as the average ejection energies. These are also given in Table I along with the area under the ELC peak obtained as described above. It is seen that the ELC process contributes $\frac{1}{3}-\frac{1}{2}$ of the total electron production cross section for these collisions.

As in the earlier work on helium, ${ }^{1}$ it is useful to compare cross sections for the same target produced by $\mathrm{H}^{0}$, $\mathrm{H}^{+}$, and $e^{-}$collisions at the same velocities. Figure 6 shows such a comparison for $150-\mathrm{keV}$ neutrals, $150-\mathrm{keV}$ protons, and $80-\mathrm{eV}$ electrons. They are shown at the same or nearly the same angles of ejection. As before, the $\mathbf{H}^{0}$ and $\mathrm{H}^{+}$cross sections are approximately equal at large ejection energies which correspond to small distances of closest approach. This is expected since for close col-

TABLE I. Values of $\sigma(W)$ in $\mathrm{m}^{2} / \mathrm{eV}, \sigma_{-}$in $\mathrm{m}^{2}$, and $W_{\mathrm{av}}$ in $\mathrm{eV}$ for $\mathrm{H}^{0}+\mathrm{H}_{2} \mathrm{O}$ collisions.

\begin{tabular}{|c|c|c|c|c|c|c|}
\hline \multicolumn{7}{|c|}{ Projectile energy $(\mathrm{keV})$} \\
\hline$W(\mathrm{eV})$ & 20 & 30 & 50 & 70 & 100 & 150 \\
\hline 2 & $4.8(-21)^{\mathrm{a}}$ & $3.9(-21)$ & $2.8(-21)$ & $2.0(-21)$ & $2.2(-21)$ & $2.2(-21)$ \\
\hline 3 & $5.1(-21)$ & $3.9(-21)$ & $2.8(-21)$ & $2.0(-21)$ & $2.1(-21)$ & $2.2(-21)$ \\
\hline 5 & $5.3(-21)$ & $4.3(-21)$ & $3.0(-21)$ & $2.1(-21)$ & $2.2(-21)$ & $2.1(-21)$ \\
\hline 8 & $5.2(-21)$ & $4.2(-21)$ & $3.2(-21)$ & $2.2(-21)$ & $1.9(-21)$ & $1.5(-21)$ \\
\hline 13 & $3.3(-21)$ & $3.5(-21)$ & $3.0(-21)$ & $2.0(-21)$ & $1.4(-21)$ & $1.0(-21)$ \\
\hline 20 & $1.6(-21)$ & $2.0(-21)$ & $2.6(-21)$ & $1.9(-21)$ & $1.3(-21)$ & $7.8(-22)$ \\
\hline 30 & $6.1(-22)$ & $8.7(-22)$ & $1.7(-21)$ & $1.7(-21)$ & $1.3(-21)$ & $7.1(-22)$ \\
\hline 50 & $1.2(-22)$ & $2.1(-22)$ & $4.9(-22)$ & $7.0(-22)$ & $1.1(-21)$ & $7.9(-22)$ \\
\hline 80 & $1.6(-23)$ & $3.3(-23)$ & $1.1(-22)$ & $1.8(-22)$ & $3.4(-22)$ & $8.8(-22)$ \\
\hline 130 & $1.6(-24)$ & $3.2(-24)$ & $1.2(-23)$ & $2.6(-23)$ & $5.8(-23)$ & $1.2(-22)$ \\
\hline 200 & $2.8(-25)$ & $4.9(-25)$ & $1.2(-24)$ & $3.0(-24)$ & $9.8(-24)$ & $2.1(-23)$ \\
\hline 300 & $6.6(-26)$ & $1.2(-25)$ & $1.2(-25)$ & $3.9(-25)$ & $9.5(-25)$ & $3.6(-24)$ \\
\hline$\sigma_{-}$ & $9.3(-20)$ & $9.6(-20)$ & $1.1(-19)$ & $9.8(-20)$ & $1.0(-19)$ & $1.0(-19)$ \\
\hline$\sigma_{-}(\mathrm{ELC})$ & $4.3(-20)$ & $3.4(-20)$ & $4.0(-20)$ & $3.2(-20)$ & $3.5(-20)$ & $4.3(-20)$ \\
\hline$W_{\mathrm{av}}$ & 13.4 & 16.5 & 23.8 & 30.1 & 38.8 & 53.1 \\
\hline
\end{tabular}

The designation $4.8(-21)$ means $4.8 \times 10^{-21}$. 
lisions the electron carried by the $\mathrm{H}^{0}$ has little effect. At lower ejection energies, the $\mathrm{H}^{0}$ cross section rises to its peak due to ELC. At still lower energies, corresponding to more distant collisions, the $\mathbf{H}^{0}$ cross sections are generally lower than the $\mathrm{H}^{+}$values due to the shielding effect of the electron. At the very lowest energies, however, the $\mathrm{H}^{0}$ cross sections again are higher. In the He paper ${ }^{1}$ it was speculated that this was due to very distant collisions in which the electron carried by the $\mathrm{H}^{0}$ caused the ionization. However, unlike the He data, the present data does not always show the similarity in shape between the $e^{-}$ and $\mathrm{H}^{0}$ data at low energy. The rise in the electron cross sections as $W \rightarrow T$ is due to scattered primary electrons. This rise, of course, corresponds to the ELC peak in the $\mathbf{H}^{0}$ curves.

\section{ACKNOWLEDGMENTS}

This paper is based upon work supported by the $\mathrm{Na}$ tional Science Foundation under Grant No. PHY-8025599. We wish to thank Kyle Hollman for help in doing the numerical integrations.
"Present address: Department of Physics, Shahid Bahonar (Kerman) University, Kerman, Iran.

${ }^{1}$ M. E. Rudd, J. S. Risley, J. Fryar, and R. G. Rolfes, Phys. Rev. A 21, 506 (1980).

2See, e.g., L. J. Puckett, G. O. Taylor, and D. W. Martin, Phys. Rev. 178, 271 (1969).

${ }^{3}$ M. M. Duncan and M. G. Menendez, Phys. Rev. A 19, 49 (1979).

${ }^{4}$ W. Meckbach, R. Vidal, P. Focke, I. B. Nemirovsky, and E. Gonzalez Lepera, Phys. Rev. Lett. 52, 621 (1984).

${ }^{5}$ D. R. Bates and G. Griffing, Proc. Phys. Soc. (London) Sect.
A 66, 961 (1953).

${ }^{6}$ A. Dalgarno and G. W. Griffing, Proc. R. Soc. Edinburgh Sect. A 248, 415 (1958).

${ }^{7}$ W. E. Wilson and L. H. Toburen, Phys. Rev. A 7, 1535 (1973).

${ }^{8}$ D. Burch, H. Wieman, and W. B. Ingalls, Phys. Rev. Lett. 30, 823 (1973).

${ }^{9}$ M. Bolorizadeh and M. E. Rudd, preceding paper, Phys. Rev. A 33, 888 (1986).

10R. D. DuBois and M. E. Rudd, J. Phys. B 8, 1474 (1975).

${ }^{11}$ A. Kövér, D. Varga, Gy. Szabó, D. Berényi, I. Kádár, S. Ricz, J. Végh, and G. Hock, J. Phys. B 16, 1017 (1983). 